\title{
Evaluation of a New Topical Treatment for Acne with Azelaic Acid-Loaded Nanoparticles
}

\author{
A. Gomes*, L. Ascensão**, P. Rijo***, M. Baptista***, S. Candeias*, N. Martinho*, A. Fernandes***, A. \\ Roberto*** and C. Reis* \\ *Universidade Lusófona (CBIOS - Laboratory of Nanoscience and Biomedical Nanotechnology (LNBN)), \\ 1749-024 Lisboa, Portugal \\ **Universidade de Lisboa, Faculdade de Ciências, Departamento de Biologia Vegetal, IBB, Centro de \\ Biotecnologia Vegetal, 1749-016 Lisboa, Portugal \\ ***Universidade Lusófona (Laboratory of Pharmacology and Therapeutics (LPT)), 1749-024 Lisboa, Portugal
}

Azelaic acid, a saturated dicarboxylic acid, is used in the treatment of acne [1,2]. However, some side effects and low patient compliance have been associated with several topical formulations of azelaic acid [3,4]. Thus, nanotechnology presents an innovative strategy that can overcome these problems [5]. Polymeric nanoparticles are generally applied to control drug release and to reduce local drug toxicity. In this study, we used the polymer PLGA (acid poly (DL-lactic-co-glycolic)) which is a biocompatible and biodegradable polymer that provides a slow and controlled drug release [6]. The aim of this study was to evaluate a new topical treatment for acne with azelaic acid-loaded PLGA nanoparticles in terms of size, surface charge, encapsulation efficiency, morphology, drug-polymer interactions, efficacy, toxicity and safety of excipients.

Nanoparticles were produced by a modified spontaneous emulsification/solvent diffusion method [7] and included into a gel of carbopol 940. Size and zeta potential were determined by photon spectroscopy and electrophoretic mobility, respectively. Efficiency encapsulation was accessed by HPLC analysis using an adaptation of a previously described method [8]. Loaded and blank nanoparticles morphology was observed by SEM. Drug-polymer interactions and thermal characteristics were evaluated by DSC. Staphylococcus epidermidis and Propionibacterium acnes susceptibility to the azelaic acid-loaded nanoparticles were tested by the broth microdilution method (efficacy test) and the minimum inhibitory concentration (MIC) values were determined using vancomycin as a positive control. The cytotoxicity of the nanoparticles was investigated on a Saccharomyces cerevisiae model [7]. Excipients safety was accessed by occluded patch tests in humans (blank nanoparticles). The tests were performed in 12 healthy, female volunteers with skin type II whose ages were in between 22-38 years. All the tests were carried out according to the Declaration of Helsinki and received the approval of local Ethical Committee.

Nanoparticles formed a soft white powder (Figure 1A). The loaded nanoparticles mean size was 378.63 \pm 60.86 $\mathrm{nm}(0.09 \pm 0.03$, P.I.) (Figure 1B) and zeta potential was $-7.82 \pm 9.01 \mathrm{mV}$. The encapsulation efficiency was $76 \pm 3.81 \%$. Entrapped nanoparticles were visible in the empty nanoparticles sample (Figure 1C). Monodispersed and spherical loaded nanoparticles were observed by SEM (Figure. 1D). System efficacy test suggested similar results of azelaic acid-loaded nanoparticles and azelaic acid in the free form against $S$. epidermidis and $P$. acnes (Table 1). DSC analysis suggested an interaction between the polymer (PLGA) and the drug. Cytotoxicity of azelaic acid-loaded nanoparticles on $S$. cerevisiae was concentration-dependent although not pronounced [7]. Occluded patch test seemed indicate that the formulation excipients were safe.

Small and anionic PLGA nanoparticles demonstrated to be efficient in the encapsulation of azelaic acid. Nanoparticles proved to be efficient against the most common bacteria associated with acne. Further studies will include additional bacteria susceptibility tests. 
References

1. Thiboutot D., J. Drugs Dermatol., 7:13-6, 2008.

2. Charnock C. et al., Eur. J. Pharm. Sci., 21:589-596, 2004.

3. Liu C.H. et al., Chem. Pharm. Bull., 60:1118-1124, 2012.

4. Amichai B. et al., J. Am. Acad. Dermatol. 54:644-646, 2006.

5. Reis C. et al., Nanomed. Nanotech. Biol. Med., 1:8-21, 2006.

6. Mainardes R. et al., Int. J. Pharm., 290:137-144, 2005.

7. Reis C. et al., submitted to Drug Dev. Ind. Pharm., 2012.

8. Ibrahiem M.M. et al., Chromatographia, 55:435-437, 2002.
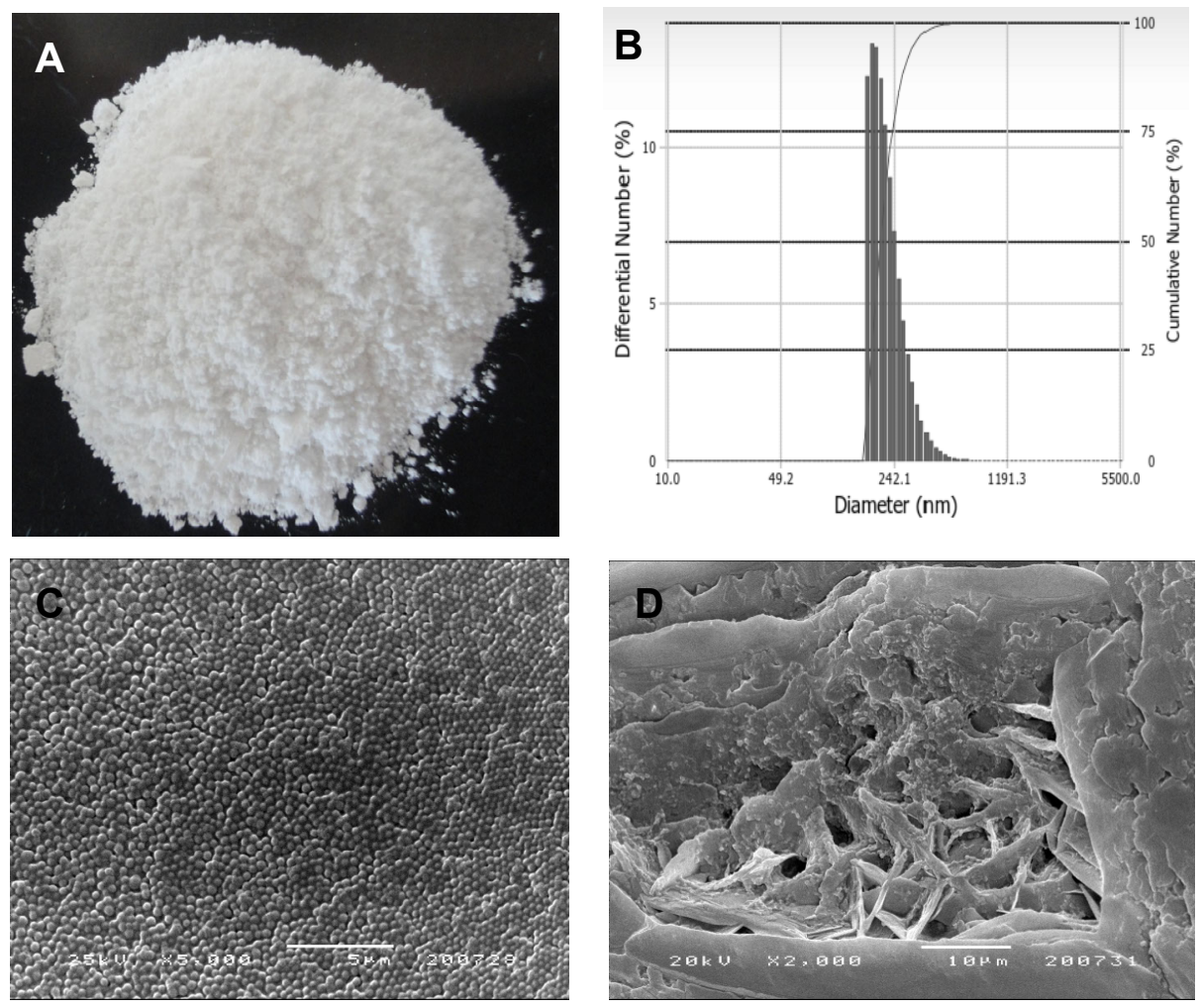

Figure 1. A) Macroscopical feature of the azelaic acid-loaded nanoparticles; B) Size particle distribution of the loaded nanoparticles; C,D) SEM micrographs of monodispersed loaded spherical particles and blank nanoparticles.

Table 1. MIC values of the tested samples at $\mathrm{pH}$ 5, against S. epidermidis and P. acnes:

\begin{tabular}{|c|c|c|c|c|}
\hline Samples & $\begin{array}{c}\text { Azelaic acid } \\
\text { MIC }\left(\mu \mathrm{g} \cdot \mathrm{mL}^{-1}\right)\end{array}$ & Azelaic acid-loaded nanoparticles & $\begin{array}{c}\text { PBS } \\
\text { MIC ( }\left(\mu \mathrm{g} \cdot \mathrm{mL}^{-1}\right)\end{array}$ & $\begin{array}{l}\text { Vancomycin } \\
\text { MIC }\left(\mu g \cdot \mathrm{mL}^{-1}\right)\end{array}$ \\
\hline S. epidermidis & 375 & 375 & $>1500$ & $<7.81$ \\
\hline P. acnes & 750 & 750 & $>1500$ & $<15.6$ \\
\hline
\end{tabular}

